\title{
The contribution of Carl Adam Petri to our understanding of 'computing'
}

\author{
Giorgio De Michelis \\ DISCo, University of Milano - Bicocca \\ gdemichedisco.unimib.it
}

\begin{abstract}
Carl Adam Petri is well known for introducing the nets having his name. It is less known that net theory was, for Petri, the kernel of a radical shift in scientific knowledge. Carl Adam Petri has not written extensively during his life and this may have been an important reason that only the most applied part of his approach, the use of nets for designing concurrent systems, has become largely popular. In this paper, I try to popularize in a larger community the radical novelty and the relevance of the approach Petri used for developing scientific knowledge of physical and social phenomena. In short, we can say that Petri's approach assumes that knowledge, even scientific knowledge, is rooted in human experience and there is no way, and no necessity, to connect it directly to an external reality.

This has, as we will see, much to do with the concept of computing and, indirectly, with the relations between science and philosophy. This paper will summarize three aspects of Petri's thinking, which deserve a wider attention: the notion of model, the new algebraic foundations for a theory of modeling (whose main example are Petri Nets) and its application to Human Communication Pragmatics.
\end{abstract}

\section{Introduction}

Carl Adam Petri (1926-2010) is well known in the Computer Science community for introducing the nets having his name that are widely studied, discussed and applied in the field of concurrent system modeling $[15,18]$. It is less known that, from its very early introduction in his $\mathrm{PhD}$ dissertation [9], net theory was, for Petri, the kernel of a radical shift in scientific knowledge. As a matter of fact, his Thesis, whose aim was discussing "the conceptual foundations of a theory of communication" $[9, \mathrm{p}$. 1], introduced a radically different approach to information and communication with respect to the widely adopted "Mathematical Theory of Communication" of Claude Shannon and Warren Weaver [17]. The differences regard not only the characterization of communication and information phenomena (we will come back on these, later in this text) but also, and mainly, some concepts that are basic in any formal model of a natural and/or social phenomenon. Petri claims, in fact, that "the representation proposed here differs from the presently known theories concerning information in at least one of the following essential points: 1 . The existence of a metric is assumed for neither space nor time nor for other physical magnitudes; 2 . Time is introduced as a strictly local relation between states; 3 . The objects of the theory are discrete, and 
they are combined and produced only by means of strictly finite techniques." [9, p. 1]. The absence of metrics, a new local notion of time, and discreteness distinguish his approach from mainstream research in natural and artificial sciences, however, this fact has neither been appreciated nor discussed, even if Petri nets gained quickly a widespread success as a means for modelling distributed systems.

Carl Adam Petri has not written extensively during his life and this may have been an important reason that only the most applied part of his approach, the use of nets for designing concurrent systems, has become largely popular. He was well aware of this fact, as it is clear from his paper "Forgotten topics of Net Theory" [13], where he discusses new and old aspects of his work, to which scholars paid too little attention.

In this paper, I want to make one small step in trying to popularize in a larger community the radical novelty and, of course, the relevance of the approach Carl Adam Petri used for developing scientific knowledge of physical and social phenomena. This has, as we will see, much to do with the concept of computing and, indirectly, with the relations between science and philosophy. The biography of Carl Adam Petri by Einar Smith recently appeared [20] is surely an important step towards filling the lack of a full and coherent presentation of the conceptual richness of his work. What I present here is, because of the limited size of this text, an incomplete account of the scientific approach of Carl Adam Petri, focusing on its relevance for philosophy of science. I do hope that younger researchers will join and surpass me in this endeavour.

This paper summarizes three aspects of Petri's thinking, which deserve a wider attention: the notion of model, the new algebraic foundations for a theory of modeling, with a survey of Petri nets basic concepts and constructs, and its application to Human Pragmatics. However a number of other important aspects of Petri's work remain untouched. Let me just mention two of them: at the mathematical level, the topological characterization of net models and, at the scientific knowledge level, the discussion of physical theories; to both of them Petri dedicated a great attention; however, space limitation does not allow me to discuss them.

\section{Modeling}

In a lecture given in June 2003 at the Petri Net conference in Eindhoven [14], as well as in several other occasions during the later phase of his life, Carl Adam Petri presented, in one or two of his slides, his viewpoint on the nature of models of natural and social phenomena, where he detaches them from any ambition to be directly related to reality. The first part of this paper is a comment on it.

The top slide (Fig. 1) poses the question: "What is modeling?" and Petri's answer claims that he prefers to the widespread view, that it is a partial function from reality to model, the view that it is a translation from a shared informal model to a formal model. 


\title{
WHAT IS MODELING?
}

\author{
Widespread view:
}

\section{REALITY ------- partial function ---- $\rightarrow$ MODEL}

\author{
but we prefer: \\ SHARED \\ FORMAL \\ INFORMAL $==$ translation $=\rightarrow$ MODEL \\ MODEL
}

Fig. 1. What is modelling? [14]

This claim introduces a radical change with respect to standard scientific approaches widely adopted, e.g., in physics, but also, frequently, in social sciences. Here modeling is characterized, without any reference to 'reality', as a way of changing the quality of what we know, from something informal, that we share but is incomplete, sometimes contradictory, and in any case rich of ambiguities, to a formal model that is, per se, fully sharable because it is reproducible (Fig. 2).

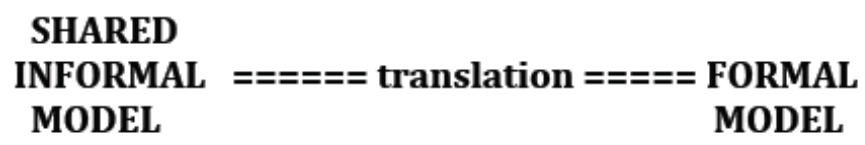

contains:

Experiences

Conventions

PLANS

Preferences

Beliefs, Illusions ?

Paradoxes? requires:

Deductive power

Share-ability

Definiteness for

Verification or

Disproof

Simple Basis

Fig. 2. Differences between standard and Petri's views on modelling [14] 
Knowledge is rooted in human experience and there is no way, and no necessity, to connect it directly to an external reality. We can relate this claim, for example, to the 'phenomenological stance' discussed by Richard Rorty [16] or to the debate raised by Bridgman operationalism in philosophy of science [4]. Knowledge, and scientific knowledge in particular, is the outcome of a process of observation, supported by technological instruments: we cannot separate the outcomes of these observations from the way we did them. This does not mean that Petri was inclined to relativism: on the contrary, he was a follower of Einstein's approach to knowledge and of his "God does not play dice" [20]. Nature, for him, is strictly deterministic, however, our knowledge cannot see it, because of the limitations of our discerning capability. In some sense, instead of considering our rationality limited by the fact that we cannot reach exact values, he claims that these values do not allow us to take into account all the elements characterizing a phenomenon (and making it deterministic): our knowledge is not an approximation of what happens in reality, rather, it is what we can get from the observations (experiments) we are doing, and therefore it is, in part, unable of avoiding a certain degree of indeterminacy. The philosophical implications of his approach are not discussed in his papers; Carl Adam Petri was not a philosopher and he always refused to discuss his work at the philosophical level: he was an engaged practitioner of scientific research and his results must be evaluated as a contribution to science, and not to philosophy!

\section{Concurrency}

From Petri's viewpoint, therefore, modeling is, as said above, a translation from informal to formal knowledge, but it cannot forget that what we know is the outcome of an observation: we should not say "this thing is...", but only "this thing appears to be...". And Petri thinks that this warning should not remain something that philosophy adds to scientific discourse, relativizing it: from his viewpoint, it should be visible in what science itself affirms, without ambiguities. For this reason, net theory avoids to use real numbers and equivalence among them in its theoretical discourse and breaks down equivalence relation (symmetric, reflexive and transitive) into two, complementary, relations: the first one (concurrency) is symmetric and reflexive, while the second one (causal order) is transitive and reflexive. In particular, concurrency is an example of a more abstract type of relation, named empirical indifference characterizing what remains of equality, if we remember that our statements (e.g.: a is b) always refer to our observations (e.g.: I can't distinguish a from b).

Intuitively, empirical indifference between the weights of two objects $\mathrm{x}$ and $\mathrm{y}$ means that they leave the pans of a beam balance in equilibrium, or, in other words, balance out each-other. Its relevance emerges, if we take seriously the question if empirical indifference is transitive or not; i.e. if $\mathrm{x}$ balancing out $\mathrm{y}$ and $\mathrm{y}$ balancing out $\mathrm{z}$, implies also that $\mathrm{x}$ balances out $\mathrm{z}$, or not. While Rudolf Carnap in his book "Einfuhrung in die Philosophie der Naturwissenschaften" (Introduction to the Philosophy of Natural Sciences; [6]) and many others are in favour of transitivity of empirical indifference, as Smith recalls [20], Petri rejects this assumption vehemently. He ar- 
gues that its transitivity cannot be justified empirically; on the contrary, it can be refuted by simple counter-examples.

Petri rejects also the position of other theorists, like Norbert Wiener [21], who, in contrast to Carnap, do recognize that the relation of directly observable empirical indifference is not transitive, but claim that there is a genuine transitive equivalence underlying it (this position has been re-proposed by Herbert Simon in his bounded rationality argument; [19]), that can be regained from the imprecise measurements by a posteriori deductions, pointing out that it is only a purely formal construction. The irreducibility of empirical indifference to equivalence is, therefore, a fundamental point distinguishing Petri's approach for it reflects the irreducible presence of the observer even in its basic mathematical structures.

We will see, in fact, that, as a relation characterized by reflexivity and symmetry, empirical indifference can have different interpretations in different fields, (concurrency is one of them) all of which are related to qualities depending on observation procedures. As an example, it has been shown that saturation of local states and closure operations on particular classes of nets [3] generate sets with an ortho-modular logical structure, like quantum logic and studies comparing nets with quantum computation have been made also by Samson Abramsky [1]. This opens an interesting field of research, investigating how nets can be used in quantum physics. Going back to the comparison between Petri discourse and traditional scientific approaches, the irreducibility of empirical indifference to equivalence questions the notion of measurement as it is characterized in science [20].

Classically, measurement is conceived as the assignment of appropriate real numbers. The objective of a measuring, in fact, is to establish the true value of a quantity.

In these approaches, observation and measurement consist in establishing and reporting the true (real) value more or less accurately. Once measured, objects may also be compared: they are equal if they have the same value. If real numbers represent the values of objects, how can we deal with them, when we only can deal with their discrete approximations? When two objects have the same value, we can always imagine that, if we do a more accurate measure, then their equality will be denied. This means that measure is intrinsically unable to give us real values. This is not a claim against measures; on the contrary, is a quest for a realistic approach to them. A more comprehensive understanding of measuring requires situating its technical procedure within its application context. In this view, the real objective of measurement appears to be providing grounds for decisions between various alternative courses of action in real-world situations. Thus the information content of a measure can be considered adequate if it allows a rational choice among the (bounded) number of possible alternatives. In this sense, every measurement generates a finite amount of information. In contrast to the classical approaches discussed above, Petri advocates the view, that in dealing with actual continuous magnitudes it is more appropriate to avoid the idealistic detour via real numbers. Rather, one should acknowledge the finiteness of the information content in measurements already in the construction of the theory. Petri suggests using "elements of a suitable chosen structure" instead of real numbers (Smith, 2014). This can be done, pragmatically, grounding any comparison on empir- 
ical observations themselves, i.e. to avoid 'equivalent' for adopting 'observably larger' and its complement 'empirically indifferent'.

\section{Petri Nets}

Petri nets (I follow, in this short presentation, the exposition line adopted by Bernardinello and colleagues in a recent paper, written for the Italian public [2]; this means that we will omit any reference to higher classes of nets, like PT nets, as well as to net processes) model systems as bi-partite graphs, constituted by nodes of two types: conditions are represented by circles, containing a mark if the corresponding condition is true, while transitions (or events) are represented by rectangles, connected through oriented arcs to their pre- and post-conditions. At any moment, the global state of the system is defined, therefore, as the set of true conditions the set of marked conditions, at that moment. The dynamic behaviour of the system can be simulated by moving the marks in the net in accordance with a simple rule: when a transition is enabled (all its pre-conditions are true, and therefore marked, and all its postconditions are false, and therefore not marked), its firing moves all the marks form its pre-conditions to its post-conditions (Fig. 3).
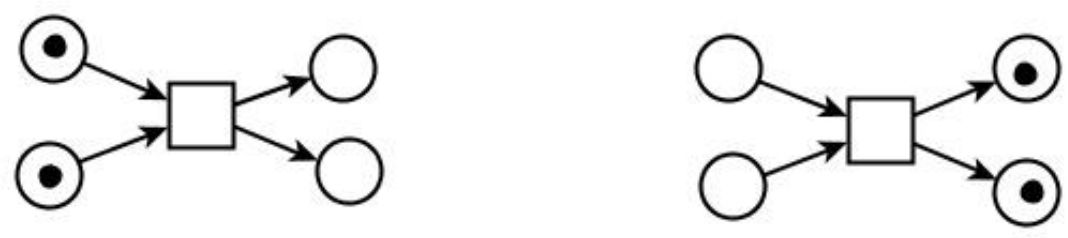

Fig. 3. Firing of a transition (before and after).

An event is, therefore, extensionally defined by its pre- and post-conditions and, if two transitions are both enabled and have fully disjoint pre- and post-conditions, then they may fire concurrently, with no reference to any temporal scale. If, on the contrary, one of the post-conditions of a transition is also pre-condition of another transition, then between the two transitions there is a causal dependency relation: the second transition can fire only after the firing of the first transition. Instead of a temporal order we have, therefore, a causal order, so that, when two or more transitions can fire at the same moment in any order, then they are causally independent, i.e. concurrent.

Two different transitions can never be simultaneous, but for when they are strictly synchronized, becoming therefore the very same transition (it is what happens when we clap: the clapping of the right hand coincides with the clapping of the left one).

It is immediate to observe that concurrency is a typical example of indistinguishability (see previous Section): in this case in fact, the observer cannot distinguish any order relation between the observed transitions. It has to be underlined that concurrency does not depend on / reflect the indeterminism of the firing of the 
transitions; rather, it is connected with the impossibility to observe a causal dependency among them: concurrency (like in-distinguishability) is a property emerging, when the discourse is about observation of facts and not facts per se.

There is a third type of relation playing a major role in nets, the relation of conflict. There is a conflict between enabled transitions (Fig. 4) when they share a precondition (forward conflict; Fig. 4, left) and when they share a post-condition (backward conflict; Fig. 4, right). When two transitions share a pre-condition, in fact, the firing of any of them will disable all the other ones, even when it is not possible to know which, among those transitions, will fire. For Petri, the correct interpretation of conflicts associates them with some lack of information: it should exist, therefore, a larger net containing new conditions (dis)solving the conflict. The solution of a conflict is, therefore, the result of flow of information to the system from its environment that is not represented in the system itself. This confirms the strictly deterministic view of Petri, who asserted it from his PhD thesis until his last works and talks, and his agreement with the views inspiring some research lines of contemporary physics.
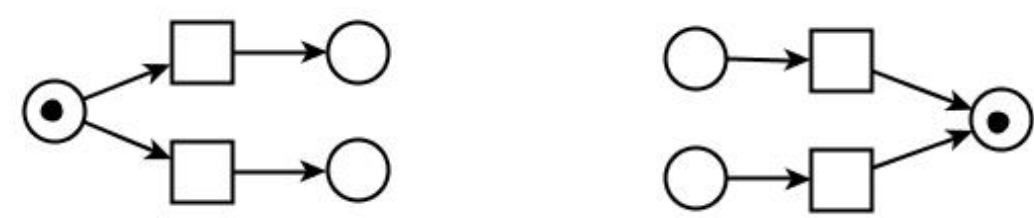

Fig. 4. Examples of forward and backward conflicts

Interpreting conflicts through information flows between a system and its environment, Petri excludes both creation and destruction of information: conflicts should be interpreted with getting new information (forward conflicts) and with loosing it (backwards conflicts). If you look at the backwards conflict at the right of Fig. 4, then you may observe that, when the token reaches the post-condition of the two transitions, then the information telling us which of them fired moving the token to the post-condition, is lost. Reversibility of behaviour is lost in net systems with conflicts: the reversibility may be restored looking at the larger net containing the conditions that dis-solve the conflicts.

Net systems allow also to present models with confusion, where confusion is characterized by a combination of a conflict between two transitions and the concurrency of one of them with another transition so that when both concurrent transitions have fired, we can not know if the conflict has been solved or not. 

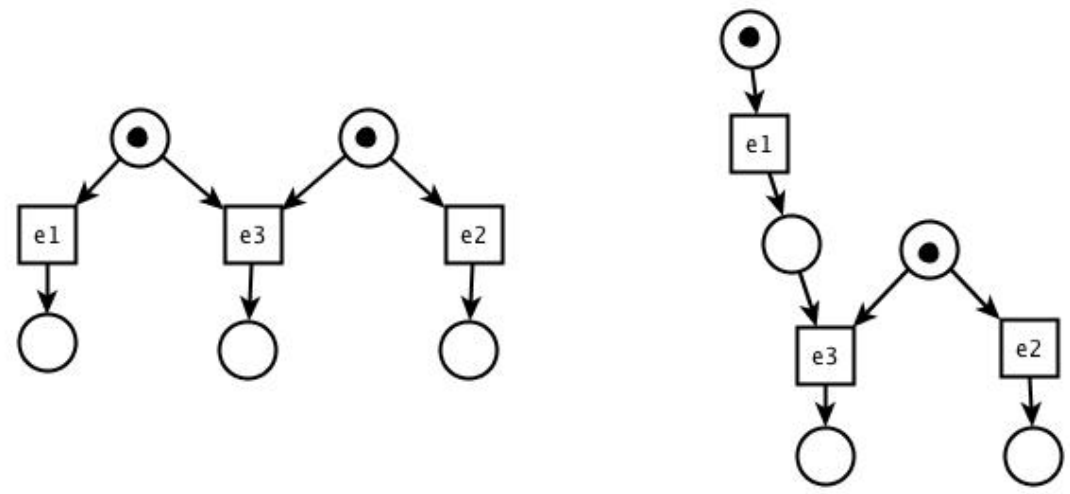

Fig. 5. Two typical confusion cases

In the net at the left of Fig. 5, for example, when e1 and e2 have fired, we do not know if their firing depends on the solution of the conflict between e1 and e 3 (liberating e2 from its conflict relation with e3) or, on the contrary, from the solution of the conflict between e 2 and e3. At the right of Fig. 5, we have a second different case of confusion, where we do not know if the concurrent firing of e1 and e 2 include the solution of the conflict between e 3 and e 2 (because the firing of e1 has generated it) or not (because e2 has fired before its conflict with e3 was generated). Confusion situations model, generally, interactions between components, whose behavioural choice are not local, so that it is not clearly specified which of them is taking decisions.

This short recall of some basic concepts of Petri nets is not aiming to offer a survey of their relevant aspects and mechanisms; rather, it aims only to show how, what we have said about modelling in the previous section, emerges with great evidence here. Not only concurrency and partial order are constitutive of nets, but also conflict and confusion are, on one hand, formulated with respect to concurrency, on the other, properties showing, even in their names, the limits of what the observer sees.

\section{Communication Disciplines}

From the early seventies, Petri paid attention to human pragmatics. For him it was a natural consequence of his interest in communication networks and computers: when, in the early seventies, computers come out of research laboratories for being used within companies and public institutions, he was trying to understand how we could figure out what they were, from the viewpoint of their users. He wanted to give an image of them that was not considering them as 'magic' machines (over-estimating their capabilities), without reducing them to the first simple applications for which they had been used (under-estimating their capabilities). Among the reasons for trying to find this well balanced image, there was also the fact that, with the passage of time, over- and under-estimations were opposed each other, without any dialogue among them. This reflection brought to a visionary schema (Fig. 6) where we can follow the 
evolution of the image or the role of the computer, as a 'convergent' oscillation towards what he considers a reasonable well balanced idea, avoiding both over- and under-estimations: the computer is becoming a "general medium for strictly organisable information flow" [10]. It means that, through email and messaging systems, for example, they do not support only message dispatching but also, and mainly, conversations. We do not have space here to discuss what this means for human practice (the interested reader may look at [7] and browse its references) but it has to be underlined that the diagram of Fig. 6 was created in the late seventies, when personal computers were still to come and coupling computers with communication required a strong visionary capability. Petri was not the unique visionary precursor of what computers became later; in particular, for his attention to their roles in shaping communication, his name has to be coupled with that of Joseph C. R. Licklider [8].

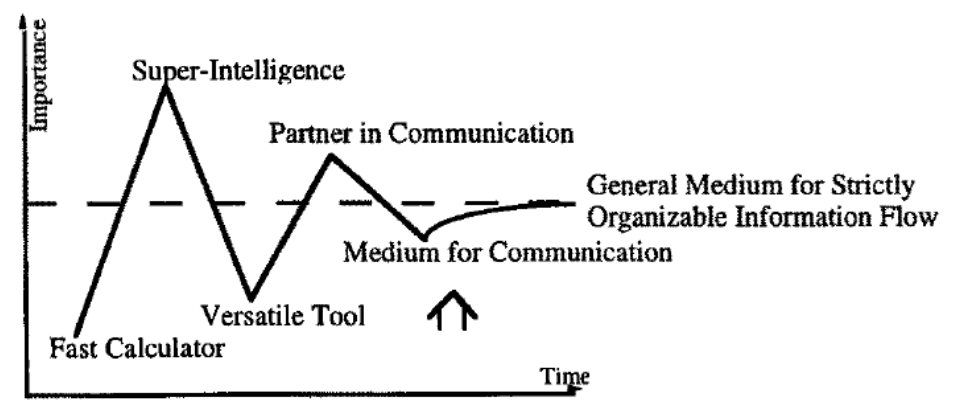

Fig. 6. The role of the computer - "Convergence" of opinions on its potential [10]

As part of his interest for human pragmatics, he introduced also Communication Disciplines [7, 10.11]. Again, this was a remarkably novel change, with respect to Mathematical Communication Theory by Shannon and Weaver [17], in viewing communication phenomena (Fig. 7).

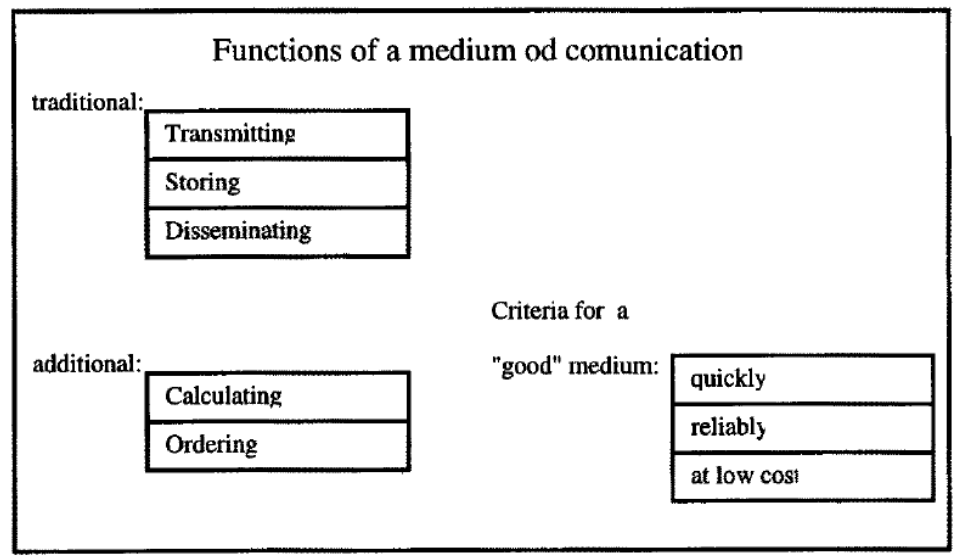

Fig. 7 - Shannon and Weaver's view on communication ([10] 
While Shannon and Weaver characterize a communication medium in terms of its functions (from the traditional ones -transmitting, storing and disseminating- to the new ones originated by the computers - calculating and ordering), Petri is interested in how humans can manage communication (Fig. 8). For this reason, he introduces a long (12) list of 'disciplines' that should be followed by a 'good' communication medium (the list is not considered by Petri complete, because innovation could request adding to it some new 'disciplines'). They streamline the flow of information within a network of humans and do not merely support information exchange. It is not by chance that Petri speaks of 'disciplines' instead of functions: communication disciplines, in fact, are disciplines both as sub-areas of a science and as constraints of behaviour.

\begin{tabular}{l}
$\begin{array}{l}\text { Functions of a medium of communication } \\
\text { ("Communication Disciplines") }\end{array}$ \\
\begin{tabular}{|l|l|}
\hline Synchronization & Identification \\
\hline Addressing & Naming \\
\hline Copying & Cancelling \\
\hline Composition & Modelling \\
\hline Authorizarion & Valuation \\
\hline Delegation & Reorganization \\
\hline
\end{tabular} \\
Criterion for a "good" medium: perform these functions \\
\hline
\end{tabular}

Fig. 8 - The Communication Disciplines [10]

Let us briefly discuss the 12 communication disciplines listed by Petri. They can be subdivided in three groups.

Those belonging to the first group (synchronisation, identification, addressing and naming) shape communication events within a network.

Synchronization is concerned with getting proper timing restraints for different activities. Net Theory has based it on a partial ordering in terms of causality as opposed to ordering in terms of time.

Identification is concerned with well-known questions such as "identify the source of a letter" and with more sophisticated problems such as proving the competence of agencies with respect to certain actions.

Addressing is concerned with describing routes or systems of paths through a net of channels and agencies.

Naming is concerned with the means through which we can understand the content of a message. 
The second (copying, cancelling and composition) and the third group (authorization, delegation, valuation and reorganization) are of a higher level and define roles of people within the network.

More specifically, the second group contains the disciplines shaping what people with a given role can do with messages.

Copying and cancelling are concerned, respectively, with rules for copying and cancelling messages and/or their attachments.

Composition is concerned with rules for creating new messages/attachments composing previous messages/attachments.

The third group contains rules for managing the network.

Authorization is concerned with access rights, scheduling obligations and supervision rules.

Delegation is concerned with role attribution in the network.

Valuation is concerned with the scarcity of resources and their exchangeability. Values do not depend on individual preferences but imply organizational constraints.

Re-organization is concerned with the rules through which a system can be changed without causing failures or disasters.

The list of communication disciplines should be considered as open ended, since it depends on the network capabilities and can be extended introducing other, higher, roles of actors in the communication network.

\section{Conclusion}

As I said above, Petri refused to discuss his ideas from a philosophy of science viewpoint: he considered himself as a scientist, and his theories had to be discussed for their capacity to enlighten natural and social phenomena. Despite this posture, I think that his work should be studied by philosophers of science, since it offers a unique case of a theoretical work modifying the grounds of scientific assertions and the mathematical language through which they are formulated. My resumé of his work is incomplete, however if it instigates the interest of the readers and motivates them to learn more about Petri's work, then I am satisfied.

\section{Acknowledgements}

What I have written in these pages is only my responsibility. I want to acknowledge the conversations I had with Carl Adam Petri, who was, patiently and effectively, answering my questions. I am also grateful to my colleagues Lucia Pomello and Luca Bernardinello with whom I did research on Nets and discussed frequently issues raised in this paper. They also reviewed carefully an initial draft of this text. Finally, I am grateful to Grzregorz Rozenberg for the advice he gave me after reading a first version of his paper. 


\section{References}

1. Abramsky, S. Petri Nets, Discrete Physics, and Distributed Quantum Computation. In: Degano, P., De Nicola, R., Meseguer, J. (eds.) (2008) Concurrency, Graphs and Models: Essays Dedicated to Ugo Montanari on the Occasion of his 65th Birthday, LNCS 5065, Springer Verlag, Berlin, 527-543 (2008).

2. Bernardinello, L., Cardone, F. and L. Pomello L'eredità di Carl Adam Petri: dagli automi alla comunicazione, Mondo Digitale, XV(62), http://mondodigitale.aicanet.net/20161/articoli/01_leredita_di_carl_adam_petri.pdf (2016).

3. Bernardinello, L., Ferigato, C. and L. Pomello, An algebraic model of observable properties in distributed systems, Theoretical Computer Science 290(1), 637-668, (2003).

4. Bridgman, P. W. The Logic of Modern Physics. Macmillan, New York, NY (1927).

5. Cardone, F., Computers and the Mechanics of Communication Outline of a Vision from the Work of Petri and Holt. In: A Beckmann, V. Mitrana, M. Soskova, (eds.) (1927) Evolving Computability. LNCS Vol. 9136, Springer Verlag, Berlin, 3-12 (1927).

6. Carnap R. Einführung in die philosophie der Naturwissenschaften. Nimphenburg Verlag, Ullstein-Frankfurt, (1986).

7. De Michelis, G. and C. Ellis, Computer Supported Cooperative Work and Petri Nets. In: W. Reisig, G. Rozenberg (eds.) Lectures on Petri Nets. II Applications, LNCS 1492, Springer Verlag, Berlin, 125-153 (1998).

8. Licklider J. C. R. and R. W. Taylor The Computer as a Communication Device. Science and Technology, 76(4), 21-31 (1968).

9. Petri, C. A. Kommunikation mit Automaten. Rheinisch-Westfaelisches Institut fuer Instrumentelle Mathematik and der Universitaet Bonn, Schrift Nr. 2. Also: Communication with Automata, Griffiss Air Force Base, New York, RADC-TR-65-377, Vol. 1, Suppl. 1, 1966 (English Translation) (1962).

10. Petri, C. A., 1977. Communication Disciplines. In: B. Shaw (Ed.), Computing System Design. Proc. of the Joint IBM University of Newcastle upon Tyne Seminar, Sep. 1976, University of Newcastle upon Tyne, 171-183.

11. Petri, C. A. Modelling as a Communication Discipline. In: H. Beilner, E. Gelenbe (eds.) Measuring, Modelling and Evaluating Computer Systems. North Holland, Amsterdam, 435-449 (1977).

12. Petri, C. A. State-Transition Structures in Physics and in Computation. Int. Journal of Theoretical Physics, 21(12), 979-992 (1982).

13. Petri, C. A. "Forgotten" Topics of Net Theory. In: Brauer, W., Reisig, W., Rozenberg, G. (eds.). Petri Nets: Applications and Relationships to Other Models of Concurrency, Advances in Petri Nets 1986, Part II, LNCS 255, Springer-Verlag, Berlin, 500-514, (1987).

14. Petri, C. A. Net Modelling - Fit for Science? Lecture presented at the $24^{\text {th }}$ European Conference on Application and Theory of Petri Nets, Eindhoven (2003).

15. Reisig, W. Understanding Petri Nets. Springer-Verlag, Berlin (2013).

16. Rorty, R., Philosophy and the Mirror of Nature. Princeton University Press, Princeton.

17. Shannon, C. E. \& W. Weaver, 1949. The Mathematical Theory of Communication. The University of Illinois Press, Urbana IL (1979).

18. Silva, M. Half a century after Carl Adam Petri's Ph.D. thesis: A perspective on the field. Annual Reviews in Control, 37, 191-219, (2013).

19. Simon, H. Bounded Rationality and Organizational Learning. Organization Science 2 (1), 125-134 (1991).

20. Smith, E. Carl Adam Petri - Life and Science. Springer Verlag, Berlin (2015). 
21. Wiener, N. A new Theory of Measurement:a study in the Logic of Mathematics. Proc. London Math. Soc. (1921) s2-19 (1): 181-205 (1919). 